\title{
Percepción del riesgo mediante sus atributos psicosociales en trabajadores de la industria metalmecánica en la ciudad de Bogotá, D.C (Colombia).
}

\author{
Perception of the risk through its psychosocial attributes in workers of the \\ metalmechanical industry in the city of Bogotá, D.C (Colombia).
}

Álvaro Fajardo Zapataㅁ, Jenny Fabiola Hernández Niño², Yuri Lilian González Valencia ${ }^{3}$, Héctor Andrés Hernández4, Myriam Leonor Torres Pérez

\section{Resumen}

Introducción. La percepción del riesgo en el ámbito laboral se convierte en un elemento crucial para entender las prácticas de trabajo inseguras de los trabajadores, teniendo en cuenta no solo las variables cognitivas sino también las psicosociales. Objetivo. Describir la percepción del riesgo mediante sus atributos psicosociales en trabajadores de la industria metalmecánica en la ciudad de Bogotá. Materiales y métodos. Estudio descriptivo transversal realizado en la ciudad de Bogotá, D.C, a través de una encuesta aplicada a los participantes del estudio. Resultados. La aplicación de la Guía NTP 578 a los trabajadores del sector metalmecánico permitió la realización del perfil comparativo de la magnitud del riesgo percibido y sus atributos para cada una de las actividades que se evaluaron. Conclusiones. Los trabajadores del sector metalmecánico, se sienten más vulnerables a las consecuencias que pueden generarles el uso de herramientas cortantes, la exposición al ruido y la exposición a gases y vapores, y le temen a la gravedad del dańo que se puede derivar estos riesgos a los cuales están sometidos en su diario vivir en su entorno laboral.

Palabras claves: riesgo, percepción, industria metalmecánica, psicosocial, laboral.

\footnotetext{
1. Médico Cirujano. Ingeniero Industrial. Candidato a Doctor en Ciencias de la Educación. M.sC en Toxicología. M.Sc en Salud Pública y Desarrollo Social. Especialista en Salud Ocupacional. Profesor Asociado del Departamento de Ingeniería y Ciencia Básicas de la Fundación Universitaria del Área Andina de Bogotá. Bogotá, Colombia.

ORCID: http://orcid.org/0000-0001-6067-3053

2. Bacterióloga. PhD en Salud Pública. M.Sc en Microbiología. Especialista en Docencia Universitaria. Líder Nacional de Investigación. Profesora de la Universidad Nacional a Distancia - UNAD, Bogotá. Bogotá, Colombia.

ORCID: http://orcid.org/0000-0003-0497-5422

3. Psicóloga. M.Sc en Salud y Seguridad en el Trabajo. Líder del programa de Tecnología en Salud y Seguridad en el Trabajo. Profesora de la Universidad Nacional a Distancia - UNAD, Bogotá. Bogotá, Colombia.

ORCID: http://orcid.org/0000-0001-5625-7508

4. Ingeniero Industrial. M.Sc en Educación Ambiental y Desarrollo Sostenible. Profesor de la Universidad Nacional a Distancia - UNAD, Bogotá.

Bogotá, Colombia.
ORCID: http://orcid.org/0000-0002-8925-3009

5. Optómetra. PhD en salud Pública. M.Sc en Evaluación en Educación, Especialista en Gerencia en Instituciones de Educación Superior. Decana de

la Facultad de la Escuela de Salud de la Universidad Nacional a Distancia - UNAD, Bogotá.

Bogotá, Colombia.

ORCID: http://orcid.org/0000-0003-2923-5754
} 


\section{Abstract}

Introduction. The perception of risk in the workplace becomes a crucial element to understand the unsafe work practices of workers, taking into account not only the cognitive variables but also the psychosocial ones. Objective. To describe the perception of risk through its psychosocial attributes in workers of the metal-mechanic industry in the city of Bogotá. Materials and methods: A cross-sectional descriptive study conducted in the city of Bogotá, D.C, through a survey applied to the study participants. Results. The application of the Guide NTP 578 to the workers of the metalworking sector allowed the realization of the comparative profile of the magnitude of the perceived risk and its attributes for each of the activities that were evaluated. Conclusions. Workers in the metalworking sector feel more vulnerable to the consequences that can result from the use of sharp tools, exposure to noise and exposure to gases and vapors, and fear the seriousness of the damage that can be derived from these risks to which they are subjected in their daily life in their work environment.

Keywords: risk, perception, metal-mechanic industry, psychosocial, employee.

\section{Introducción}

La percepción del riesgo como proceso cognitivo y social en los seres humanos puede afectar la forma como las personas interactúan con los diversos peligros en su actividad laboral, considerándolos como poco dañinos para su salud, además de creer tener el control y el conocimiento sobre ellos, lo que podría llevar a desencadenar un accidente laboral $(1,2)$.

En este contexto, Morillejo plantea que la percepción del riesgo en el ámbito laboral se convierte en un elemento crucial para entender las prácticas de trabajo inseguras de los trabajadores, teniendo en cuenta no solo las variables cognitivas sino también las psicosociales. Lo cual indica que este proceso está bajo la influencia de los conocimientos previos, los patrones culturales y factores del medio en el que las personas se desenvuelven. De este modo el autor expone que la conducta preventiva de las personas está en función de la amenaza percibida y de los beneficios que pueda recibir por llevarla a cabo. A su vez esta percepción depende de la susceptibilidad y de la gravedad de las consecuencias que los sujetos perciben al experimentar un accidente laboral, por lo que un trabajador tomará medidas de prevención dependiendo de lo vulnerable o no que se perciba (3).

Slovic, en sus estudios concluye que conociendo el riesgo percibido por las personas, se podrían anticipar muchos de los comportamientos riesgosos que son adquiridos y determinar el conocimiento que tienen las personas sobre el riesgo al que se enfrentan, es fundamental para poder intervenir en ellos (4).

Este mismo autor, en sus investigaciones encontró que los riesgos que son fácilmente aceptados están asociados a actividades vistas como altamente beneficiosas para la perso- 
nas. En este sentido se podría considerar que muchas de las condiciones inseguras a nivel laboral pueden ser toleradas por el trabajador y vistas como parte inevitable del mismo, partiendo de los beneficios encontrados de su actividad, como la remuneración económica, el estatus social, etc. (5).

La misma situación es planteada por Blanco et al, cuando exponen que en la toma de decisiones, la persona tenderá a aquella que le genere mayor utilidad. Así se podría llegar a plantear que un trabajador se enfrentará a una situación riesgosa más fácilmente si de la misma, obtendrá algún beneficio, por ejemplo mayor remuneración, ahorro de tiempo, aceptabilidad social, etc. (6).

Dee, en un estudio de percepción del riesgo en el sector agrícola encontró que los trabajadores reconocen que su labor tiene altos riesgos. Sin embargo, consideran que gracias a la realización de esta actividad ellos obtienen calidad de vida para sí mismos y su familia. Así, los beneficios alcanzados se logran por medio de la aceptación de los riesgos, minimizando los mismos, frente a los beneficios recibidos por la realización de la labor. Este autor concluye en su investigación, que la percepción del riesgo es el resultado de un análisis costo beneficio que se basa en el conocimiento subjetivo de las personas el cual está influenciado por condiciones políticas, económicas y culturales (7).

En este sentido Puy, argumenta que los criterios utilizados por las personas para valorar la magnitud del riesgo atienden a dimensiones psicológicas, sociales y culturales que trascienden a considerar la probabilidad de causar daño o muerte (8). Arezes et al. encontraron que los trabajadores no consideraban los altos niveles de ruido como peligroso para su salud y por lo tanto no juzgaban necesario el uso de protectores auditivos durante la realización de su labor, con lo cual sustenta que la percepción del riesgo debe ser considerada como un tema central en el diseño e implementación de los programas para la conservación de la salud, indicando que entender la manera en que los trabajadores perciben el riesgo es fundamental para una mejor gestión del riesgo (9).

Estos estudios evidencian la importancia de reconocer la influencia de la percepción del riesgo sobre las conductas de evitación a los mismos, y plantean la pertinencia de describir los atributos del riesgo percibido con el fin de identificar cuáles de ellos presentan una correlación positiva con respecto a la valoración de la magnitud percibida.

La industria metalmecánica comprende diferentes actividades de manufactura que utilizan productos de la siderurgia o sus derivados, con el fin de realizar su transformación, ensamble o reparación. De igual forma, esta industria se relaciona con las empresas de las ramas electromecánicas y electrónicas. Los trabajadores de este sector económico se encuentran expuestos a condiciones de trabajo peligrosas como el manejo de herramientas cortantes, máquinas que trabajan a rápidas revoluciones y con altos voltajes, lo que lo convierte en una industria altamente propensa a accidentes de trabajo. Con lo expuesto es necesario el diseño de estrategias orientadas a la minimización de estos riesgos en los puestos de trabajo. En este sentido, la Seguridad y Salud en el Trabajo es un área de importancia en este tipo de industrias, debido a las gran- 
des implicaciones que tienen las condiciones de trabajo en la salud de las personas y a la vez en las pérdidas económicas y humanas en una organización $(10,11,12,13)$.

Según la Agencia Europea para la Salud y Seguridad en el Trabajo, las organizaciones tienen el propósito de aumentar al máximo el rendimiento de sus recursos y mejorar la productividad, para el incremento de las utilidades financieras y, desde luego, el crecimiento en el nivel de vida de los colaboradores de la empresa, lo anterior afirma la necesidad de generar estrategias concernientes a conservar la integridad de vida de las personas en su lugar de trabajo (14).

En el reporte de accidentalidad en Colombia de 2014 emitido por el ministerio de Trabajo, se observa que dentro de los primeros sectores de mayor tasa de accidentalidad, enfermedad y número de muertes se encuentran en los sectores de minas y canteras, agricultura, industria manufacturera, administración pública y hoteles y restaurantes (15).

Para generar las estrategias apropiadas de gestión del riesgo, es imprescindible conocer la percepción del riesgo que tienen los trabajadores frente a los peligros que se exponen diariamente en su medio laboral.

\section{Materiales y métodos}

Estudio descriptivo transversal. Se realizó un muestreo aleatorio por localidad. Se aplicó el instrumento NTP 578(16): Riesgo percibido: un procedimiento de evaluación del Instituto Nacional de Seguridad e higiene en el trabajo de Espańa, que examina las características del riesgo disgregadas por los siguientes
Ítems: conocimiento científico disponible; conocimiento por parte del sujeto expuesto; novedad/familiaridad; efecto demorado de las consecuencias; voluntariedad de la exposición; controlabilidad/evitabilidad; letalidad de las consecuencias; temor que produce y potencial catastrófico. Se tomó como error el cinco por ciento $(5 \%)$, y un nivel de confianza del $95 \%$. El tamaño de la muestra se estimó en 855 encuestas. La información fue recogida entre febrero y marzo de 2017 , en 16 de las 20 localidades de Bogotá, en donde se agrupan las industrias del sector metalmecánico. El muestreo fue realizado por un grupo de estudiantes, quienes fueron previamente capacitados. Las variables investigadas fueron: edad, género, experiencia en el oficio y formación académica y los ítems referidos de la norma NTP 578. Se realizó una estimación cuantitativa de la magnitud del riesgo y valoración de los atributos del riesgo percibido. No se realizó ninguna intervención durante el desarrollo del estudio. Los análisis se realizaron en SPSS. El estudio fue sometido al comité de ética de la Universidad Nacional a Distancia - UNAD.

\section{Resultados}

En el estudio participaron 855 trabajadores el sector metalmecánico, donde la edad mínima fue de 17 años y la máxima de 97 ańos; un promedio de edad de 35,45 años; una desviación estándar de 11,73 años. La moda correspondió a 27 años y la mediana a 34 años.

El 33\% (282) de los trabajadores que participaron en el estudio tenían edades comprendidas entre los 26 y 35 años, un 24,8\% (212) entre 36 a 45 años, un 21,3\% (182) entre 18 y 25 años, un 14,2\% (122) entre 46 a 55 años; los hombres mayores de 55 ańos correspondie- 
ron al 6,2\% (53), y el restante 0,5\% (4) eran menores de 18 ańos. El 82\% (701) de los trabajadores que participaron en el estudio fueron hombres y el 18\% (154) mujeres:

En cuanto a la formación académica de los trabajadores del sector metalmecánico, el 33,8\% (289) tenían el bachillerato completo, un $14,9 \%$ (127) el bachillerato incompleto, un 13\% (111) habían terminado estudios técnicos, un 6,7\% (57) manifestaron que habían terminado una carrera universitaria, mientras que un 5,8\% (50) tenían estudios tecnológicos completos; un porcentaje igual manifestó que no había terminado los estudios técnicos. El 5,5\% (47) refirió que no había terminado la primaria, mientras que el 5,1\% (44) manifestó que únicamente había terminado sus estudios primarios. Finalmente, un 1,5\% (13) de los trabajadores refirió estudios completos de posgrado y un $0,5 \%$ (4) expreso que no tenía ninguna clase se estudios.

Con relación a la experiencia en el oficio del sector metalmecánico, el 39,8\% (341) manifestó tener entre 1 y 5 ańos, el 17,2\% (147) menos de un año, un 17,1\% (146) entre 11 a 20 años, un 16,6\% (142) entre 6 a 10 años. Quienes tenían experiencia laboral en el ramo entre 21 a 30 años correspondieron al 6,8\% (58) y finalmente un 2,5\% (21) manifestaron tener más de 30 años en el oficio.

Gráfico 1. Tendencia de las medias de los riesgos evaluados.

Tendencia de las medias de los riesgos evaluados

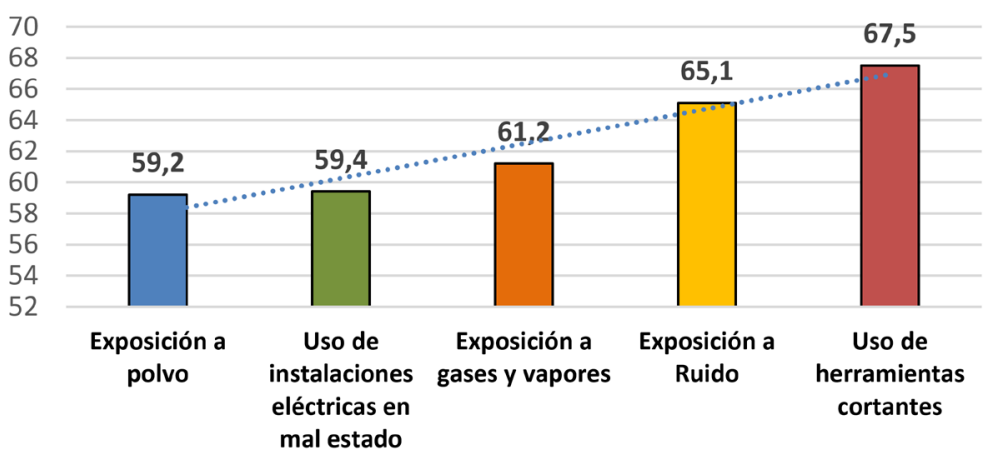

Fuente. Elaboración propia.

Para los trabajadores del sector metalmecánico que participaron en el estudio, las actividades que representaron el mayor riesgo, correspondieron al uso de herramientas cortantes $(\bar{X}=67,5$ y $S=25,6)$; seguidos por la exposición al ruido $(\bar{X}=65,1$ y $S=25,0)$; luego la exposición a gases y vapores $(\bar{X}=61,2$ y $S=26,9)$; el uso de instalaciones eléctricas en mal estado
$(\bar{X}=59,4$ y $S=28,17)$; y finalmente, la exposición a polvo con $(\bar{X}=59,2$ y $S=27,0)$.

El uso de herramientas cortantes fue la actividad peligrosa que obtuvo la más alta calificación por parte de los trabajadores, que se evidenció en el alto conocimiento acerca de los daños que puede causarles su uso $(\bar{X}=5,6$ 
y $S=1,6)$. Además, consideran que los efectos por el uso de estas herramientas en mal estado se darán en un corto tiempo ( $\bar{X}=3,8$ y $S=2,1)$.

Así mismo, manifestaron tener un conocimiento alto $(\overline{\mathrm{X}}=5,5$ y $S=1,7)$ acerca del peligro de exponerse al ruido. Así mismo consideran que el conocimiento existente sobre este factor de riesgo es alto $(\overline{\mathrm{X}}=5,5$ y $S=1,7)$ y sienten un alto temor por los riesgos que implica esta exposición $(\overline{\mathrm{X}}=5,5$ y $\mathrm{S}=1,7)$. Los trabajadores perciben como moderados los controles para prevenir los daños generados por este factor de riesgo $(\overline{\mathrm{X}}=4,7$ y $S=1,6)$.

En cuanto al uso de instalaciones eléctricas en mal estado, los trabajadores del citado sector sienten un alto temor al daño que se puede derivar por el deterioro de dichas instalaciones $(\bar{X}=5,3$ y $S=1,7)$, y perciben una baja posibilidad de daño $(\bar{X}=3,9$ y $S=1,8)$. Además, consideran que los efectos por el uso de las instalaciones eléctricas en mal estado se darán en un corto tiempo $(\overline{\mathrm{X}}=3,9$ y $S=1,8)$.

Con relación a la exposición a gases y vapores, los trabajadores del sector metalmecánico sienten un alto temor al daño que se puede derivar por la exposición a sustancias químicas $(\bar{X}=5,2$ y $S=1,7), \quad$ y además, perciben como moderados los controles para prevenir los dańos $(\overline{\mathrm{X}}=4,1$ y $S=1,7)$.

Por otra parte, los trabajadores tienen un conocimiento alto $(\overline{\mathrm{X}}=5,0$ y $S=1,7)$ acerca del peligro de exponerse al polvo, así mismo consideran que el conocimiento existente sobre este factor de riesgo es alto $(\overline{\mathrm{X}}=5,0$ y $S=1,7)$ y sienten un alto temor por los riesgos que implica esta exposición ( $\bar{X}=5,0$ y $S=1,7)$. Así mismo, perciben como moderados los controles para prevenir los daños generados por este factor de riesgo $(\overline{\mathrm{X}}=4,2$ y $S=1,7)$.

\section{Discusión}

El estudio encontró que aunque la mayoría de las personas que trabajan en este sector son hombres y están en un grupo etario que corresponde a la mitad de la vida, también trabajan personas muy jóvenes y algunas de muy avanzada edad. Se halló que existe un porcentaje muy importante de mujeres que labora en este sector de la economía.

Por otra parte, es de resaltar el nivel educativo de los trabajadores de este sector, ya que si bien el mayor porcentaje tiene estudios secundarios, también se encontró que muchos tienen formación técnica, tecnológica y profesional. Así mismo, con relación a la experiencia en el oficio en el sector metalmecánico, el estudio encontró que los trabadores participantes en este estudio manifestaron tener una gran experiencia trabajando en las labores propias de sus oficios.

El estudio permitió evaluar la percepción del riesgo en los trabajadores del sector metalmecánico, encontrando que los trabajadores de este sector se sienten vulnerables a las consecuencias que pueden generarles el uso de herramientas cortantes, la exposición al ruido y la exposición a gases y vapores, y le temen a la gravedad del daño que se puede derivar estos riesgos a los cuales están sometidos en su diario vivir en su entorno laboral. Además los trabajadores ven como alta la posibilidad de experimentar dańo como consecuencia de utilizar los elementos de trabajo en mal estado. Lo anterior coincide con el estudio de Corrales et al. (17), quienes encontraron que los trabajadores 
reconocían que el ruido podía dañar su audición; pero las conclusiones no coinciden con las de Arezes et al. y otros autores (9, 18-22), quienes en su estudios encontraron que los trabajadores no consideraban como peligrosos los altos niveles de ruido, tampoco con el de Zamorano et al. (23), quienes encontraron un bajo nivel de conocimiento en la prevención de los riesgos laborales.

Sumado a lo anterior los trabajadores del sector metalmecánico perciben que existe un conocimiento de los riesgos ocupacionales por parte de los responsables de la prevención de las empresas de este sector industrial.

Es de anotar que el estudio presentó algunas limitaciones en cuanto a la no inclusión de todas las empresas que constituyen el espectro del sector metalmecánico. Otra limitación importante la constituyó el muestreo, dado que los trabajadores que participaron en el estudio fueron exclusivamente de la ciudad, donde tal vez exista un nivel cultural más alto.

Los autores expresan los agradecimientos a los estudiantes de Universidad Nacional a Distancia UNAD quienes participaron en el diligenciamiento de las encuestas.

\section{Conflictos de intereses: Ninguno}

Fuentes de financiación: La presente investigación fue financiada por la Universidad $\mathrm{Na}$ cional a Distancia UNAD.

\section{Referencias}

1. Mann, L. Elementos de psicología social. México: Limusa, 1990.

2. Wildavsky, A. Theories of risk perception: Who fears what and why? The MIT Press on behalf of American Academy of Arts \& Sciences. 1990, 119(4): 41-60.

3. Morillejo, E. A. La percepción del riesgo en la prevención de riesgos laborales. Apuntes de Psicología. 2002, 20(3): 415-426.

4. Slovic P. Perception of risk. Science, 1987, 236(4799), 280-285.

5. Slovic, P. The perception of risk. Earthscan Publications. Londres. 2000.

6. Blanco A, Sánchez F, Carrera P CA y RD. Supuestos teóricos para un modelo psicosocial de las conductas de riesgo. En S. y. Yubero, Sida: Una visión multidisciplinar. España: Universidad Castilla, La Mancha; 2000: 41-62

7. Dee Elkind, P. Perception of risk, stressors, and locus of control influence intentions to practice safety behaviors in agriculture. Journal of Agromedicine. 2007, 12(4): 7-25.

8. Puy A. Percepción social del riesgo: Dimensiones de evaluación y predicción: Tesis Doctoral. Universidad complutense de Madrid. Departamento de Psicología.2002.

9. Arezes, PM, y Miguel AS. Individual perception of noise exposure and hearing protection in industry. Human Factors. 2005, 47(4): 683-692.

10. Servicio Nacional de Aprendizaje, Sena [Internet]. Manizales, Colombia. Caracterización ocupacional del sector metalmecánico; 2002 [citado el 20 de Julio de 2017]. Disponible en https://repositorio.sena.edu. co/bitstream/11404/2124/1/3039.pdf

11. Morelos GJ y Fontalvo T. Caracterización y análisis del riesgo laboral en la pequeña y mediana industria metalmecánica en Cartagena, Colombia. Soluciones de posgrado EIA. 2013, (10): 15-42.

12. García Y. Análisis y evaluación de la incidencia de la 
percepción de riesgos en las conductas de seguridad en el ámbito laboral. Trabajo de investigación. Universidad complutense de Madrid. 2009.

13. Instituto colombiano de normas técnicas y certificación ICONTEC. Guía para el diagnóstico de condiciones de trabajo o panorama de factores de riesgo, su identificación y valoración GTC 45. ICONTEC, 1997.

14. Agencia Europea para la Seguridad y la Salud en trabajo. [Internet]. Luxemburgo: Estudio de investigación. La responsabilidad social de las empresas y seguridad y salud en el trabajo, 2005 [citado el 25 de Julio de 2017]. Disponible en: https://docplayer. es/6844170-La-responsabilidad-social-de-las-empresas-y-la-seguridad-y-la-salud-en-el-trabajo.html

15. Ministerio de Trabajo, Mintrabajo [Internet]. Colombia: Indicadores del Sistema General de Riesgo Laboral; 2014 [citado el 17 de Agosto de 2017]. Disponible en: http://fondoriesgoslaborales.gov.co/ documents/infoestadistica/2014/INFORME\%20INDICADORES\%20SGRL\%202011\%20-\%202014. pdf

16. Ministerio de Trabajo y asuntos sociales de España [Internet]. España. NTP 578: Riesgo percibido: un procedimiento de evaluación, 2001 [citado el 22 de Julio de 2017]. Disponible en http://www.insht.es/ InshtWeb/Contenidos/Documentacion/FichasTecnicas/NTP/Ficheros/501a600/ntp_578.pdf

17. Corrales M, Tovalín H, Rodríguez M. Percepción del Riesgo Sobre Protección y Pérdida Auditiva en Trabajadores Expuestos a Ruido en el Trabajo. Ciencia \& Trabajo. 2009:1-4 http://bases.bireme.br/cgi-bin/ wxislind.exe/iah/online/?IsisScript=iah/iah.xis\&s$\mathrm{rc}=$ google $\&$ base $=$ LILACS \&lang $=\mathrm{p} \&$ nextAction $=1-$ nk\&exprSearch $=523032 \&$ indexSearch=ID

18. Bedoya, E. Atención de urgencias en Cartagena, Colombia. NOVA. 2017; 15 (27): 91 - 101

19. Gómez, E., Rodríguez, A., Ordosgoitia, K., Rojas, M., Severiche, C. Riesgos psicosociales en personal de asistencia de una clínica de tercer nivel de la ciudad de Cartagena de Indias en 2016. NOVA. 2017;
15 (27): $77-89$

20. Ibarra, A., Rua E. Evaluación de la calidad en la atención al usuario del servicio de urgencias del hospital público de Yopal en Casanare, Colombia. NOVA. 2018; 16 (30): 21-31

21. Moreno, S., Lorena Chaparro, L., Criado, L. Vega, O., Cuenca, I. Magnitud de efecto de un programa dirigido a cuidadores familiares de personas con enfermedad crónica. NOVA. 2018; 16 (29): 11-20

22. Sierra, D., Bedoya, E. Prevalencia de hipoacusia neurosensorial inducida por ruido en empresas del sector madera de la ciudad de Cartagena. 2015. NOVA. 2016; 14 (26): 47-56

23. Zamorano B, Parra V, Peña F, Castillo Y. Conocimiento y actitud en prevención de trabajadores lesionados de una empresa metalmecánica en México. Salud de los Trabajadores, 2009, 17(1): 49-57. http://www. insht.es/InshtWeb/Contenidos/Documentacion/TextosOnline/ProyectoSubvencionados/2007/2007\%20 Resumen\%20final\%20RISKS.pdf 